\title{
The Color Application of the Representative Pop Art in Modern Design — Illustrated by the Case of MAOS Design ${ }^{i}$
}

\author{
JI Qian ${ }^{1}$, ZHENG Jiayu ${ }^{1, a}$ \\ ${ }^{1}$ Huazhong University of Science and Technology,430074 Wuhan
}

\begin{abstract}
This study uses Kuler tools to extract color from representative traditional Pop Art works. A total of 25 color samples have been extracted and put into the HSB model for position distribution analysis, while 70 color samples have been extracted from the Pop style modern design works. Through analyzing and contrasting the color samples of traditional pop art and modern pop art design work, the author has summarized the similarities and differences in order to provide new ideas for color research and application.
\end{abstract}

\section{Overview of Pop Art style}

Pop Art was born in the British art form in the mid1950s, and Pop Art is also called neo-realism [1]. Pop is often regarded as a "popular, fashionable" acronym, so it represents a popular culture.

The characteristics of Pop style are mainly in the following three aspects [2]:

(1) Pursuing popularity and popular interest, emphasizing novelty and uniqueness in design, and adopting strong color processing.

(2) Pop style is not a simple and consistent style, but a mixture of various styles from the perspective of design.

(3) The pursuit of unique and rarity, it's constantly changing.

\section{Color characteristics of Andy Warhol and Roy Lichtenstein's Pop Art works}

Andy Warhol is the initiator and main advocator of the American Pop Art Movement and has made a unique contribution to the development of Pop Art [3]. (Figure $1)$.

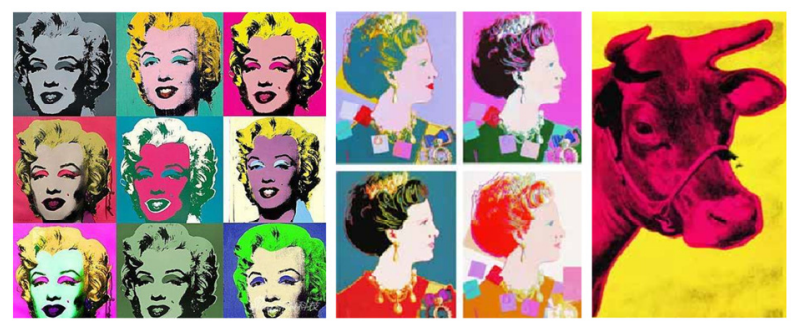

Figure 1. The representative work of Andy Warhol. left: Marilyn Diptych, middle: Queen Elizabeth, Right: Cattle

As a superstar of Pop Art, Roy Lichtenstein has promoted a new era in the artistic expression of
American painting. His works represent his humorous life [4]. (Figure 2).

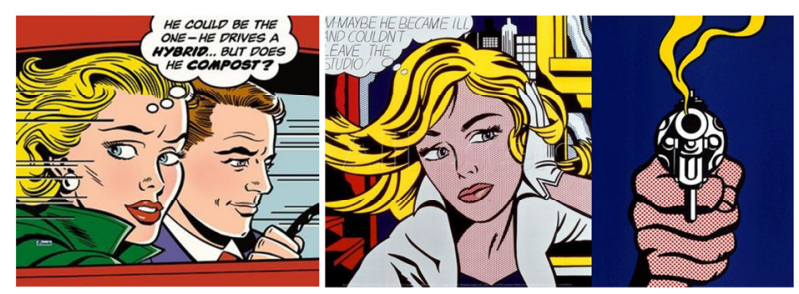

Figure 2. The representative work of Roy Lichtenstein. In the Car (1963 painting)

Photoshop and Kuler is the main color extraction tools of the research. Andy Warhol's representative work Marilyn Monroe has been used as a trial exploration for color extraction. One of the method is to import the image into Photoshop and save it in web format to get the main colors. Photoshop extracts the colors, but also black strokes and gray shadows, however they are not the target research object in this paper, so Photoshop tools is not convenient for the study of the color. While through method 2, the image will be imported into Kuler, select the color scene button for extraction, there are 6 modes of color scene, including colorful, light, soft, deep, dark and customization, so method 2 is more suitable for the research. Figure 3 shows the Photoshop extraction color interface, and Figure 4 is the Kuler extraction color interface.

\footnotetext{
* Corresponding author: ${ }^{a} 1104508815 @ q q . c o m$
} 


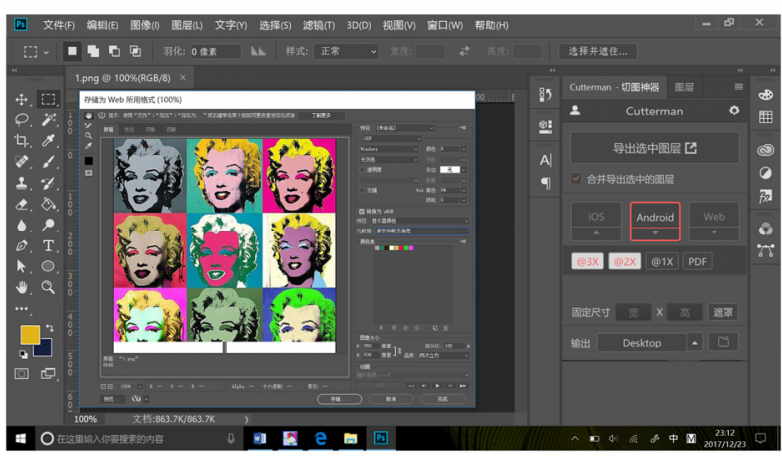

Figure 3. Photoshop extraction color interface

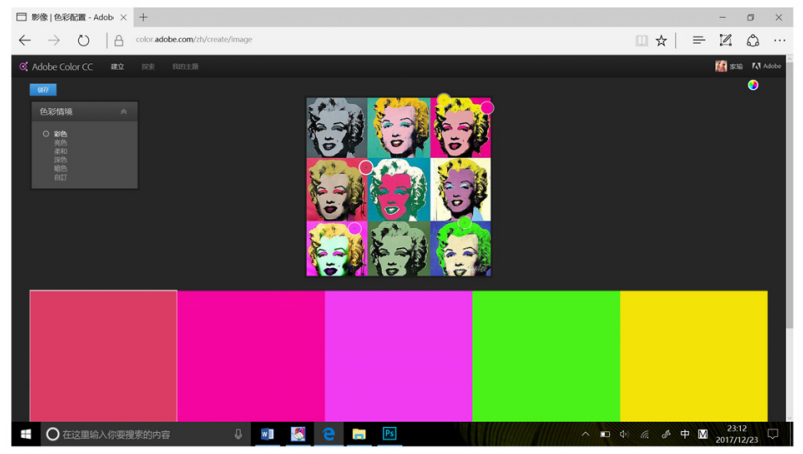

Figure 4. Kuler extraction color interface.

Kuler has been used to select the "colorful" mode for color extraction, and the color extraction analysis of the representative works of the two Pop Art leader figures can objectively summarize the color characteristics of Pop Art. Figure 5 is the color extraction by using Kuler.

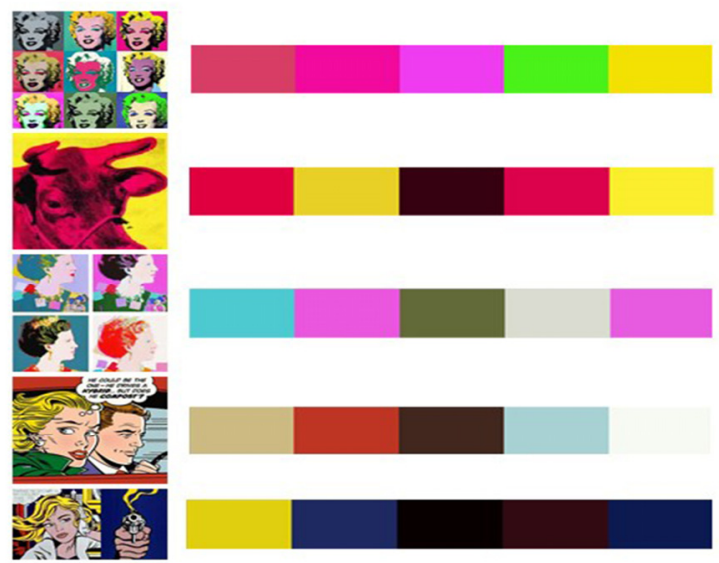

Figure 5. Color extraction by using Kuler

The color samples are extracted from the HSB value using a Photoshop color-sucking tool. The HSB is a color mode based on the human eye, where $\mathrm{H}$ represents the hue, $\mathrm{S}$ represents the saturation, and $\mathrm{B}$ represents the brightness [5]. Figure 6 is the HSB color model.

(1) Hue: In the standard color ring of $0 \sim 360^{\circ}$, according to the angle value identification. For example, red is $0^{\circ}$ and orange is $30^{\circ}$ [6].

(2) Saturation: refers to the intensity or purity of the color. Saturation represents the proportion of color components in hue, measured as a percentage from $0 \%$ (gray) to $100 \%$ (fully saturated). Saturation on the colored facade gradually increases from inside to outside [7].

(3) Brightness: It is the degree of lightness and darkness of the color. It is usually measured from the percentage of 0 (black) to $100 \%$ (white). It gradually decreases from the top to the bottom in the color facade. The upper edge is $100 \%$, the bottom line is $0 \%$ [8].

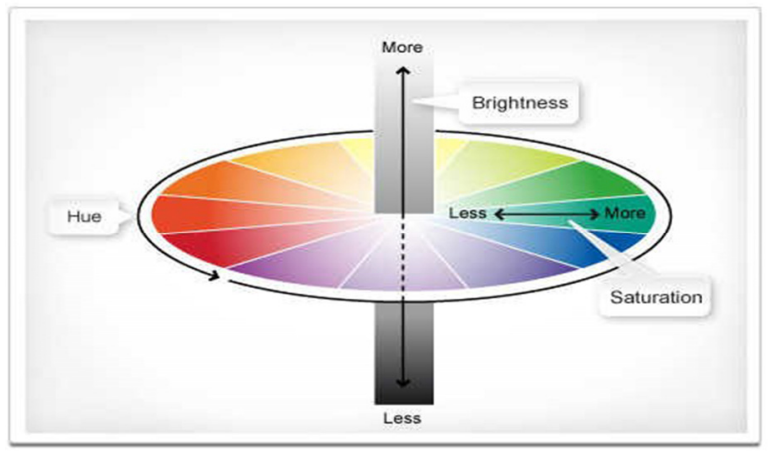

Figure 6. HSB color model

The extraction and analysis of the HSB values will provide the clues to obtain the main potential color rules of the representative Pop Art works. Figure 7 shows the HSB colors of pop art works.

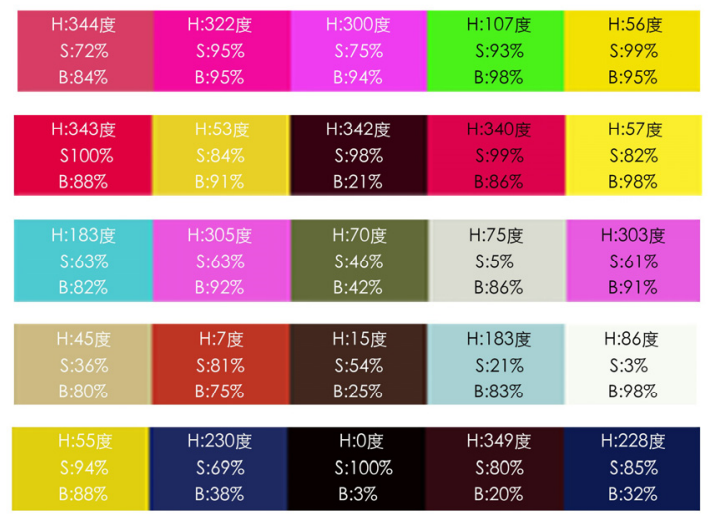

Figure 7. HSB colors of pop art works

Put the extracted color samples into the HSB model to get the color distribution of representative Pop Art works. Figure 8 shows the HSB color distribution map of them. According to the above analysis, there are 25 color samples extracted from the Pop Art representative works. The $B$ is on the left side of the map and the $H$ and $S$ are on the right side of the map.

In the left $\mathrm{B}$ distribution chart, there are 17 out of the 25 sample points being located between $80 \%$ and $100 \%$, accounting for $0.68 ; 7$ out of the 25 sample points between $20 \%$ and $80 \%$, accounting for 0.28 ; only 1 sample point between $0 \%$ and $20 \%$, accounting for 0.04 . Therefore, the brightness is higher in the above representative works of Pop art, the brightness is mainly above $80 \%$.

In the right $\mathrm{H}$ distribution chart, there are 9 samples between 0 to $90^{\circ}$, accounting for $0.36 ; 3$ samples between 90 to $180^{\circ}$, accounting for 0.12 ; 3 samples between 180 to $270^{\circ}$, accounting for $0.12 ; 10$ samples between $270 \sim 360^{\circ}$, accounting for 0.4 . Therefor in the above representative works of pop art design in the distribution of red, orange and purple more. 
In the right $\mathrm{S}$ distribution chart, there are 2 samples between $0 \% \sim 20 \%$, accounting for $0.08 ; 2$ samples between $20 \% \sim 40 \%$, accounting for $0.08 ; 3$ samples between $40 \% \sim 60 \%$, accounting for $0.12 ; 8$ samples between $60 \% \sim 80 \%$, accounting for $0.32 ; 10$ samples between $80 \% \sim 100 \%$, accounting for 0.4 . Therefore, in the above pop art representative design samples, the saturation ratio is the mainly among $80 \%$ to $100 \%$, and $72 \%$ of the samples are distributed between $60 \%$ and $100 \%$ of saturation; The saturation of the representative design works of pop art is high, and the saturation of most samples is above $60 \%$.

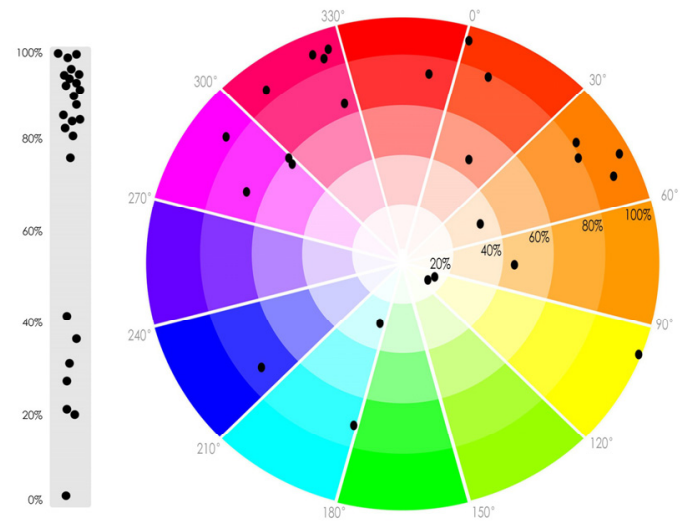

Figure 8. Pop Art representative works HSB color distribution.

The left represents the sample brightness (B), the right represents the sample hue $(\mathrm{H})$ and saturation $(\mathrm{S})$

\section{The color application of representive Pop Art works in modern design}

The style of Pop Art applications in modern design is numerous and extensive. The use of Pop Art style in graphic design can make people feel relaxed and pleased and have strong infectivity, can disseminate information while bringing people and art closer together [9]. MAOS is chosen to analyse the color of representative popular culture communication as an example in order to find the features of the modern pop style.

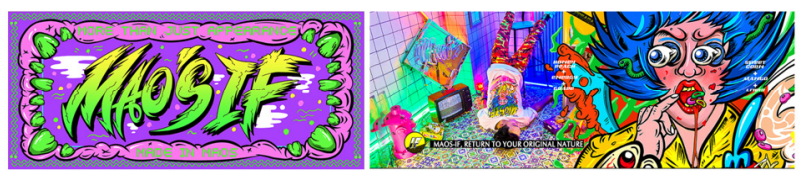

Figure 9. Works of MAOS

It is a cultural media company founded in Beijing by independent designer Mao Ting in 2015. The company's main production projects are brand image packaging, stage vision and visual image packaging of concert, party, TV, video platforms and online programs, such as "Sweet Talk", "Summer Sweetheart", "College students are here.", "Big vs. Trump", "The Temptation of Dinner" and "1.3 Billion Decibels". The influence of the program is very extensive. The main audiences of these programs are young people, they prefer the bold visual style. In the spread of popular culture, their works have subverted the entertainment industry's perception of visual packaging of variety shows; they are diverse in style and easy to attract attention. Figure 9 is a work of a seemingly cultural studio. In this section, color has been extracted and analysed of the two series of works designed by MAOS through the same methods.

The program with the most attention is the "Qipa Shuo" which is the first Chinese speech talent show created by Iqiyi in 2014. Its total hits have broken 100 million, and the amount of microblog topic readings also easily breaks the 1 billion marks. Attracted a large number of fans after $80 \mathrm{~s}$ and $90 \mathrm{~s}$, figure 10 shows the color design of QiPaShuo. The designer said "pink is a candy color, on behalf of fun, cute and young; purple represents romantic, dreamy and authoritative; ice green is a symbol of fresh and clean sunlight. [10]" Those are 3 main colors that are commonly used in the show.

Seven graphic visual designs based on different scenes and themes for the posters of QiPaShuo have been analyzed to explore the color of the visual design, Figure 10 shows the color extraction of visual design.

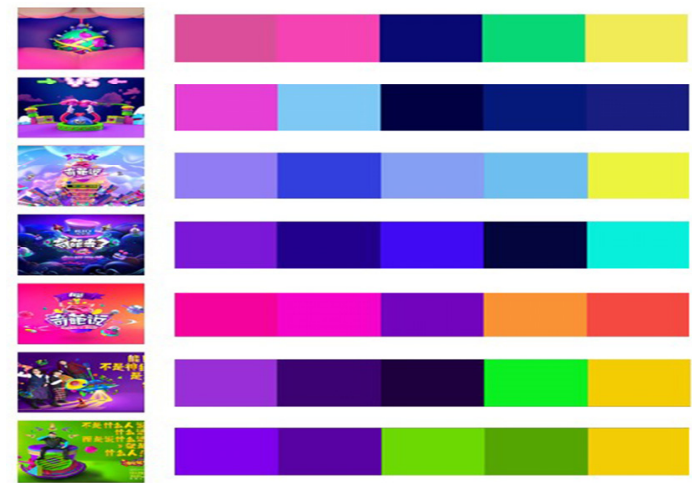

Figure 10. Color extraction of QiPaShuo

In addition to the visual communication for the show, it also involves the product packaging design. Figure 11 shows that the color of packaging design for KFC, which reflects the youth's passion and trend is very bold.

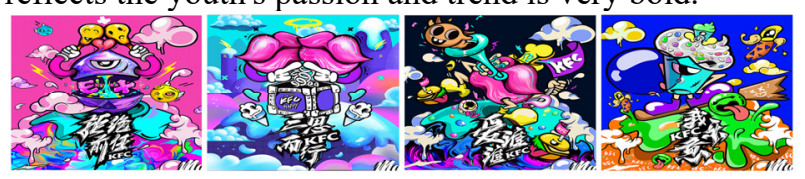

Figure 11. KFC packaging design

Figure 12 shows the visual color extraction of KFC packaging.

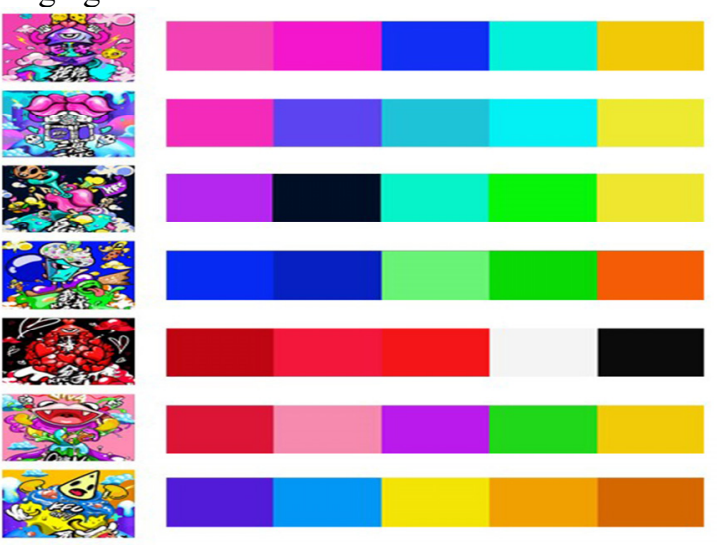

Figure 12. Color extraction of KFC

Using Photoshop to demonstrate the HSB values of the colors in QiPaShuo and KFC, Figure 13 shows the 
distribution of extracted colors in the HSB model, figure 14 is the HSB color distribution map. The series of works extracted above have a total of 70 color samples.

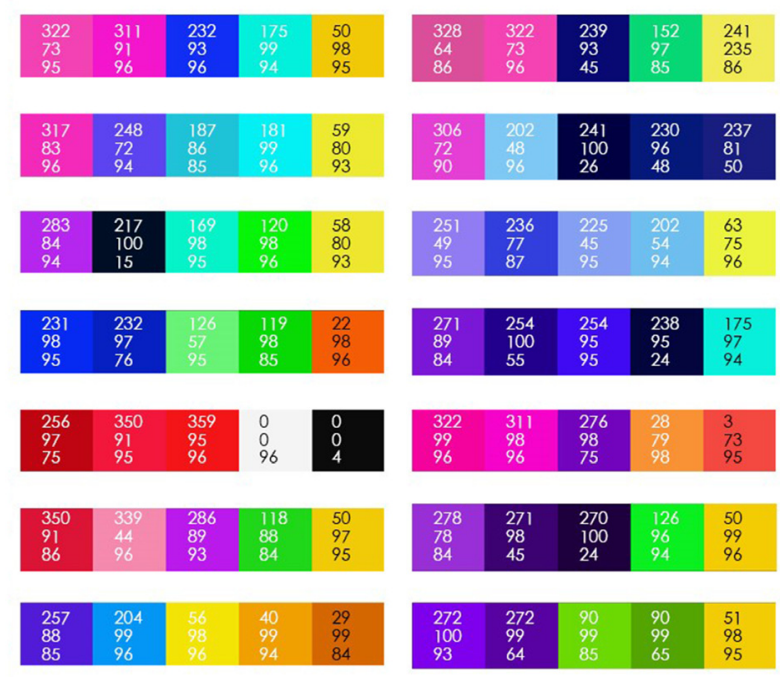

Figure 13. HSB value of QiPaShuo and KFC
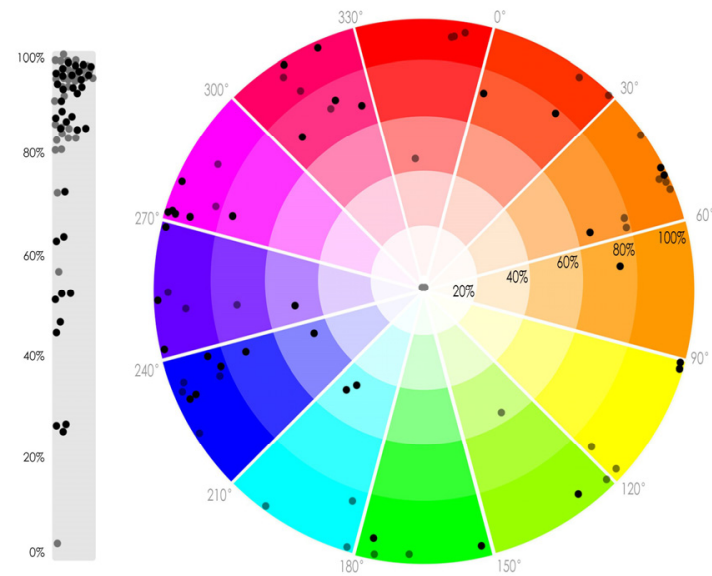

Figure 14. Color distribution in HSB of MAOS works. The left represents the sample brightness (B), the right represents the sample hue $(\mathrm{H})$ and saturation $(\mathrm{S})$

It shows that the color samples are mainly distributed among $80 \% \sim 100 \%$ in the saturation distribution chart, and mainly concentrated in the range of $70 \% \sim 100 \%$ in brightness ratio distribution chart. Hue is larger in blue, purple and red, and other color samples are evenly distributed in hue.

\section{Conclusion and outlook}

The color brightness and saturation of Pop Art works are very high. The modern Pop Art works are more colorful than the initial Pop Art works, and the collocations are more diverse (Figure 15).

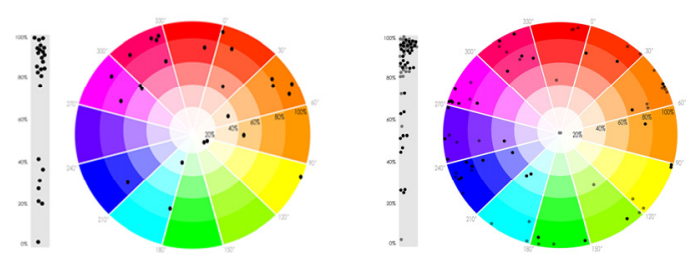

Figure 15. Color Distribution of Pop Art and modern works

This paper mainly discusses the distribution of color usage of Pop Art and modern design, Kuler is used to extract the colors in the works, and then analyzes the color samples through HSB to obtain the HSB color distribution map. However, this paper does not match the color of pop art and the analysis of the proportion of color in the screen is analyzed. How high saturation of the color will make the picture more harmonious and unified is the content of the follow-up discussion of the research, so as to deeply understand the color matching of Pop Art and get the principle of gorgeous matching instead of tacky collocation.

\section{Acknowledgment}

Supported by National Natural Science Foundation of China (Grant No: 51708236 )

Supported by Fundamental Research Funds for the Central Universities (Grant No: 2016YXMS273 )

\section{References}

1. L.Y. Li. Pop Art. M. People's Fine Arts Publishing House (2008)

2. X.L. Yin. Links between Fine Arts and Design from the Viewpoint Pop Art. J. Decoration, 2003(1):105105.

3. M.T. Jiang. Pop Art Research by Andy Warhol. D. Anhui University of Finance and Economics, 2015.

4. Y.Liu, H. Liu. Behind the Image: Roy Lichtenstein Pop Art and Influence. J. Fine Arts, 2014(2).

5. G.S. Huang. Color Design. M. China Textile Press, 2001.

6. X.M. He, D.Q. Lu, J.L. Lu, et al. Research on the Color HSB Images of Women's Shoes. J. Packaging Engineering, 2017(14): 174-178.

7. L. Qu, N. Wang, G.Y. Wu, et al. Vehicle License Plate Location Based on Color Saturation and Character Features. J. Computer Technology and Development, 2003, 13(9):6-7.

8. H.Y. Wang, J. Shao, Y. Gao, et al. Color Design of Helmet Aiming Interface. J. Electro-optics \& Control, 2016(6):64-67.

9. M.J. Hu. The Application of Pop Art in Modern Decorative Art: Taking Graphic Design as an Example. J. Art Education Research, 2017(13). 
10. Y.Y. Chen. "QiPaShuo"-Rational Carnival under the Background of Visual Culture Communication. J . Journal Research Guide, 2015(11). 